\title{
microRNA response element-regulated TIKI2 expression suppresses the tumorigencity of malignant gliomas
}

\author{
CHUAN-YOU LI ${ }^{1}$, HONG-YUN ZHANG ${ }^{1}$ and YI-LU GAO ${ }^{2}$ \\ ${ }^{1}$ Department of Brain Surgery, Xinxiang Center Hospital, Xinxiang, Henan 453000; ${ }^{2}$ Department of Neurosurgery, \\ The Affiliated Hospital of Nantong University, Nantong, Jiangsu 226001, P.R. China
}

Received December 3, 2013; Accepted May 28, 2014

DOI: $10.3892 / \mathrm{mmr} .2014 .2412$

\begin{abstract}
Glioma is the most common brain malignancy and has a very poor prognosis. The current treatment options have a minimal benefit on prolonging patient survival time. Accumulating data have shown that the WNT signaling pathway has a critical function in the progression and invasion of glioma. Thus, targeting WNT signaling appears to be an effective anti-glioma strategy. TIKI 2 was recently found to suppress the activation of the WNT signaling pathway by post-translationally modifying secreted WNT proteins. The implication of TIKI2 aberrance in cancers and its potential therapeutic effect, however, has not been studied. In the present study, a glioma-specific adenoviral vector was constructed, which was regulated by response elements of $m i R-124$, to express TIKI2 in glioma cells (Ad-TIKI2-124). Ad-TIKI2-124 was found to potently suppress the activation of WNT signaling in glioma cells. This inhibitory effect on the WNT signaling pathway lead to the reduction in proliferation, colony formation ability and invasion of glioma cell lines. In addition, animal experiments confirmed that the expression of the Ad-TIKI2-124 construct could compromise the tumorigenicity of glioma cells in vivo. Furthermore, this glioma-selective TIKI2 expression protected normal cells from toxicity. In conclusion, the present study demonstrated that adenovirus-mediated TIKI2 therapy may be used for glioma treatment and therefore warrants further clinical studies.
\end{abstract}

\section{Introduction}

Malignant glioma is the most common brain tumor, with a high ability of invasion, metastasis and recurrence and poor prognosis (1). Current treatment options, including surgery, chemotherapy and radiotherapy, have undesirable outcomes, and therefore, novel therapeutic strategies are required (2).

Correspondence to: Dr Chuan-You Li, Department of Brain Surgery, Xinxiang Center Hospital, 16 Jinsui Road, Xinxiang, Henan 453000, P.R. China

E-mail: chuanyoulixx@gmail.com

Key words: glioma, TIKI2, WNT, miR-124, adenovirus
Gene therapy has been shown to be an alternative, efficient therapeutic strategy for glioma treatment. Tumor suppressor genes, such as p53, and small hairpin (sh)RNA for oncogenes, can be delivered into glioma cells $(3,4)$.

The WNT/ $\beta$-catenin pathway has been well documented to be essential for the proliferation, survival, metastasis and neoangiogenesis of glioma cells (5). The WNT gene family consists of 19 members, which are soluble ligands for the cellular transmembranal receptors Frizzled and low density lipoprotein receptor-related protein (6). For glioma, WNT5a and WNT7b (particularly WNT5a) are overexpressed and secreted into the extracellular space in an autocrine or paracrine manner (6). Subsequently, WNT proteins activate downstream signaling through $\beta$-catenin-dependent and -independent mechanisms. The activation of the WNT/ $\beta$-catenin pathway ultimately regulates cell proliferation, motility and tumorigenicity of glioma cells (7). Decreased expression of WNT inhibitors is a predominant mechanism causing ectopic activation of WNT signaling in glioma. Epigenetic silencing of WNT pathway inhibitor genes by supermethylation of their promoters accounts for the enhanced activation of WNT signaling in certain cases of glioma (8). A study using $\beta$-catenin small interfering (si) RNA demonstrated that targeting the WNT pathway reduces glioma growth (9). Suppression of the WNT pathway therefore appears to be an effective anti-glioma strategy.

The TIKI gene family of proteases have been previously identified as a new class of WNT inhibitors, which utilize a distinct mechanism to negatively regulate the activation of WNT signaling (10). The human transcript has two TIKI orthologs, TIKI-1 and TIKI-2. TIKIs have three separate functional domains: i) Signal peptide sequence; ii) TIKI domain; and iii) transmembrane domain. The TIKI domain is highly conserved between different organisms and exerts the predominant protease activity for WNTs. This can result in the removal of several N-terminal amino acids from the mature WNT proteins, or its immature form in the endoplasmic reticulum (ER), thereby inducing oligomerization of WNT proteins and minimizing their activity. siRNA-mediated TIKI2 downregulation potentiates the WNT-3a-induced WNT pathway in mammalian cells. Overexpression of TIKI proteins has been shown to be able to suppress the WNT/ $\beta$-catenin pathway (10). This indicated that adenoviral vector-based TIKI expression may be an effective method to treat glioma. However, to date, 
the association of TIKIs with glioma and the effect of TIKI gene therapy for glioma have remained to be investigated.

In the present study, miRNA response elements (MREs) of tumor suppressor $m i R-124$, which have previously caused reduced expression levels in a number of glioma cells (11), were constructed in an adenoviral vector with glioma specificity. Subsequently, this vector was used to express TIKI2 to suppress the aberrant WNT activation in glioma cells, aiming to impair the proliferation, invasion and tumorigenesis of glioma cells both in vitro and in vivo.

\section{Materials and methods}

Cell lines. Human glioma, U-87 MG, U-251 MG, U-373 MG and M059J, neuronal, $\mathrm{HCN}-2$, fibroblast, MRC-5 and BJ, and endothelial, HUV-EC-C, cell lines were purchased from the American Type Culture Collection (ATCC, Manassas, VA, USA). Human L-02 normal liver cells were obtained from the Shanghai Cell Collection (Shanghai, China). Cells were cultured using recommended media supplemented with $10 \%$ of fetal bovine serum (FBS; Invitrogen Life Technologies, Carlsbad, CA, USA), $4 \mathrm{~mm}$ glutamine, 100 units $/ \mathrm{ml}$ penicillin, and $100 \mu \mathrm{g} / \mathrm{ml}$ streptomycin in a $5 \% \mathrm{CO}_{2}$ and humidified atmosphere at $37^{\circ} \mathrm{C}$. Media included the following: ATCC-formulated Eagle's Minimum Essential Medium for U-87 MG, U-251 MG, U-373 MG, MRC-5 and BJ; Dulbecco's modified Eagle's medium (DMEM)/Ham's F12 medium (1:1) for M059J; DMEM for HCN-2; ATCC-formulated of F-12K Medium for HUV-EC-C; and RPMI-1640 for L-02 (All media were purchased from Invitrogen Life Technologies)

Primary culture. For primary astrocyte culture, cell samples were obtained from aborted fetuses with informed consent from the pregnant females, according to the previously published procedures approved by the Ethical Review Board of Xinxiang Center Hospital (Xinxiang, China). Briefly, brain tissue from the anterior fontanelle was dissected into pieces with the removal of meninges, followed by trypsin digestion. The digested cells were filtered using a steel net and cultured in DMEM supplemented with 15\% FBS. Glial fibrillary acidic protein (GFAP) expression was confirmed by immunofluorescent staining.

Quantitative polymerase chain reaction ( $q P C R)$. Fresh cancerous and noncancerous brain tissues $(\mathrm{n}=9)$ were obtained with written informed consent from all patients according to protocols approved by Ethical Review Board in Xinxiang Center Hospital (Xinxiang, China), in order to analyze miR-124 expression. All patients underwent surgical resection of primary glioma at Xinxiang Center Hospital (Xinxiang, China).

Total RNA was extracted from glioma and noncancerous brain tissue as well as from the indicated cell lines using TRIzol $^{\text {TM }}$ solution (Sigma-Aldrich, St Louis, MO, USA). The reverse transcription reaction was performed using the TaqMan $^{\circledR}$ MicroRNA Reverse Transcription kit (Applied Biosystems, Carlsbad, CA, USA) following the manufacturer's instructions. qPCR was performed using TaqMan ${ }^{\circledR}$ 2X Universal PCR Master Mix (Applied Biosystems) on a CFX96 ${ }^{\mathrm{TM}}$ Real-Time PCR Detection System (Bio-Rad
Laboratories, Hercules, CA, USA) supplied with LightCycler Data Analysis software, version 3.5 (Roche Diagnostics, Basel, Switzerland).

To determine the abundance of TIKI2 mRNA in adenovirus (Ad)-infected cells, a multiplicity of infection (MOI) of 10 of the indicated adenoviruses were added to the cell cultures. Following $48 \mathrm{~h}$, cells were harvested for RNA extraction, followed by reverse transcription into cDNAs using Rever Tra Ace qPCR RT Kit (Toyobo, Osaka, Japan) according to the manufacturer's instructions. The 7.5- $\mu \mathrm{l}$ reactions were incubated for $30 \mathrm{~min}$ at $16^{\circ} \mathrm{C}, 30 \mathrm{~min}$ at $42^{\circ} \mathrm{C}, 5 \mathrm{~min}$ at $85^{\circ} \mathrm{C}$ and then held at $4^{\circ} \mathrm{C}$. qPCR was performed using TaqMan ${ }^{\circledR} 2 \mathrm{x}$ Universal PCR Master Mix (Applied Biosystems) using a CFX96 ${ }^{\mathrm{TM}}$ Real-Time PCR Detection System (Bio-Rad Laboratories) supplied with analytical software. The following primers were used: TIKI2, forward 5'-GACCTGCGTGCTGATC-3' and reverse 5'-TAAAAGAAGATGACAG-3'; Axin 2, forward 5'-CCGGTGGACCAAGTCCTTAC-3' and reverse 5'-TCCATTGCAGGCAAACCAGA-3'; Lgr5, forward 5'-TGAACACCTGCTTGATGGCT-3' and reverse 5'-TGCTGCGATGACCCCAATTA-3'. The housekeeping gene was GAPDH, forward 5'-TCAGTGGTGGACCTG ACCTG-3'; reverse 5'-TGCTGTAGCCAAATT CGTTG-3'.

Luciferase reporter assay. The sense and antisense sequences containing four copies of the miR-124 MREs were synthesized, annealed and inserted into the XhoI and NotI sites of psiCheck2 vectors (Promega Corp., Madison, WI, USA) immediately following the Renilla luciferase gene, in order to construct an MRE-regulated luciferase reporter, namely psiCheck2-124. The inserted MRE sequence for the miRNAs used in the present study was as follows: 5'-TCGAGGATATCACAAACACCGTGCCTTAAACAAACA CCGTGCCTTAAACAAACACCGTGCCTTAAACAAACA CCGTGCCTTAAACAAACACCGATATCGC-3'; 5'-GGCC GCGATATCGGTGTTTGTTTAAGGCACGGTGTTTGT TTAAGGCACGGTGTTTGTTTAAGGCACGGTGTTTGT TTAAGGCACGGTGTTTGTGATATCC-3'. Italicization indicates endonuclease site.

Following $48 \mathrm{~h}$ of transfection of U-87 MG, U-251 MG, astrocyte, MRC-5 and L-02 cells, the cells were harvested and treated with lysis buffer. Firefly and Renilla luciferase activities were determined with using the Dual-Luciferase reporter system (Promega Corp.) following the manufacturers instructions.

Adenovirus construction. Adenovirus Ad-enhanced green fluorescent protein (EGFP) was kindly provided by Dr. Zhao (General Hospital of Chengdu Military Area Command of Chinese PLA, Chengdu, China). Ad-TIKI2 was constructed using the procedures described below. The sequence of the human TIKI2 gene used in this study was available from GenBank (accession no., JQ653416). The cDNA templates were obtained from HeLa cells. A set of primers was used for PCR-based TIKI2 gene amplification: Forward 5'-GCCGTCGACACCATGCACGCCGCCCTG-3' and reverse 5'-GCCGATATCTCAGGAGGGCCCAA-3'. The PCR product was digested with SalI and EcoRV restriction endonucleases and then inserted into the pShuttle-cytomegalovirus (CMV) vector at the same site, to 
become pShuttle-CMV-TIKI2. For the construction of the pShuttle-CMV-TIKI2-124, the DNA fragment containing four copies of the miR-124 MREs was released by digestion with EcoRV from the psiCheck2-124 vector, followed by insertion into the same site of the pShuttle-CMV-TIKI2 to generate the pShuttle-CMV-TIKI2-124 construct.

The pShuttle-CMV-TIKI2 and pShuttle-CMV-TIKI2-124 constructs were co-transfected using pAdEasy into human HEK-293 embryonic kidney cells with Lipofectamine 2000 transfection reagent (Invitrogen Life Technologies). Following three rounds of plaque purification and PCR-based identification, the adenoviruses (Ad-TIKI2 and Ad-TIKI2-124) were harvested and purified by $\mathrm{CsCl}$ gradient centrifugation. The titers of the adenoviruses were determined using the TCID $_{50}$ method (12) in HEK-293 cells and shown as plaque-forming units per milliliter (pfu/ml).

Immunoblotting assay. To determine the expression levels of phosphorylated and total $\beta$-catenin in adenovirus-infected cells, 10 MOIs of the indicated adenoviruses were added to the cell cultures. After $48 \mathrm{~h}$, the proteins were harvested using an M-PER ${ }^{\circledR}$ Mammalian Protein Extraction Reagent (Thermo Fisher Scientific, Waltham, MA, USA), separated by polyacrylamide gel electrophoresis and transferred onto $0.45 \mu \mathrm{m}$ nitrocellulose membranes. The membranes were then blocked with $5 \%$ fat-free dry milk for $2 \mathrm{~h}$. The membrane was incubated with monoclonal rabbit IgG against $\mathrm{p}-\beta$-catenin $(1: 1,000), \beta$-catenin $(1: 1,000)$ and GAPDH $(1: 2,000)$ primary antibodies (Cell Signaling Technology, Inc., Danvers, MA, USA) for $2 \mathrm{~h}$, and then incubated overnight with the corresponding secondary antibody (polyclonal goat IgG against rabbit IgG, 1:10,000; ZSGB-BIO, Beijing, China). The membranes were visualized using SuperSignal West Dura Extended Duration Substrate (Thermo Fisher Scientific) and the ChemiDoc $\mathrm{XRS}^{+}$chemiluminescence imaging system (Bio-Rad Laboratories).

$T$ Cell Factor (TCF) reporter assay. The activation of endogenous WNT signaling was further examined by a TCF reporter dual luciferase assay. Glioma cells were seeded in 24-well tissue culture plates and transfected with the TCF reporter vector (TOPflash) (Millipore, Billercia, MA, USA) and control Renilla luciferase reporter vector (pRL-TK) (40:1) (Promega Corportation), using Lipofectamine 2000 (Invitrogen Life Technologies). Overnight, cells were infected with the indicated adenoviruses of 10 MOI. Forty-eight hours following adenovirus administration, the cells were collected and resuspended in lysis buffer (Promega Corp.) and the levels of luciferase activity were monitored for the adenovirus and Renilla expression levels using the Dual-Luciferase Reporter Assay kit and a luminometer (Promega Corp.) according to the manufacturer's instructions. All experiments were performed in triplicate.

MTT assay. Adenoviral vectors of indicated MOIs were added to the cell cultures. Following transduction for the indicated times, $50 \mathrm{ml}$ MTT $(1 \mathrm{mg} / \mathrm{ml})$ was added. The MTT reagent was removed after $4 \mathrm{~h}$ and $150 \mathrm{ml}$ dimethyl sulfoxide was added. The spectrophotometric absorbance was measured on a model 550 microplate reader (Bio-Rad Laboratories) at
$570 \mathrm{~nm}$, with a reference wavelength of $655 \mathrm{~nm}$. Cell viability was calculated according to the following formula: Cell viability $=$ absorbance value of infected cells/absorbance value of uninfected control cells.

Apoptosis analysis by cytometry. The cells were transduced with Ad-TIKI2, Ad-TIKI2-124 or Ad-EGFP of $10 \mathrm{MOI}$ for $48 \mathrm{~h}$. The cells were then harvested and fixed in $70 \%$ ethanol and stained with propidium iodide (PI; $200 \mathrm{mg} / \mathrm{ml}$ ) for cytometrical analysis using an Aria II sorter (BD Biosciences, San Diego, CA, USA). For each group, 10,000 cells were counted to determine the percentages of the sub- $G_{0} / G_{1}$ population.

Contact-independent colony formation assay. To determine the contact-independent colony formation efficiency, cells were seeded into low-attachment 96-well plates (Corning Life Sciences, Cambridge, MA, USA) at a density of 10 cells per well. The cells were infected with $10 \mathrm{MOI}$ of Ad-EGFP, Ad-TIKI2 or Ad-TIKI2-124 and maintained in stem cell medium. Equal volumes of fresh media were replaced to replenish the growth factors and nutrients every three days. Methyl cellulose (1\%) (Sigma-Aldrich) was added to prevent cell aggregation. In this way, an individual sphere derived from a single cell was produced. Cells were incubated for 14 days and then the number of wells which contained spheres with a diameter $>75 \mu \mathrm{m}$ were counted. The experiments were performed in triplicate.

Transwell ${ }^{\circledR}$ assay. The effect of Ad-TIKI2 and Ad-TIKI2-124 on the invasion of glioma cells was evaluated using the Transwell ${ }^{\circledR}$ assay in a 24 -well plate (Corning Life Sciences). The Transwell membrane with $8.0 \mu \mathrm{m}$ pores was coated with or without Matrigel ${ }^{\circledR}(1 \mu \mathrm{g} / \mu \mathrm{l}$; BD Biosciences). Cells were infected with $10 \mathrm{MOI}$ of adenoviruses and resuspended in DMEM/F12 basic medium. The cells were then seeded in the upper chambers $\left(5 \times 10^{4}\right.$ cells each). The nutritional attractant in the lower chambers was DMEM/F12 medium containing $10 \%$ FBS. Subsequently, the cells were allowed to migrate for $24 \mathrm{~h}$. The migrant cells on the membranes of the lower chambers were stained with crystal violet and then the cells in $\geq 10$ randomly selected microscopic fields at 100x magnification were counted and expressed as the invasive cells per microscopic field. All the experiments were performed in triplicate.

Animal experiments. The procedures for animal experiments were approved by the Committee for the Use and Care on Animals in the Xinxiang Center Hospital (Xinxiang, China).

A U-87 MG tumor xenograft model was established by injecting $2 \times 10^{6}$ cells into the right flanks of five-week-old male BALB/c nude mice (obtained from the Shanghai Laboratory Animal Center, Shanghai, China) The mice were kept at an $18-22^{\circ} \mathrm{C}$ aired condition with $50-60 \%$ humidity. As soon as tumors grew to $6-8 \mathrm{~mm}$ in diameter, 24 mice were randomly and equally divided into four groups $(n=6)$. The mice were intratumorally injected with $100 \mu$ l phosphate-buffered saline (PBS), with or without $2 \times 10^{8}$ pfu of Ad-EGFP, Ad-TIKI2 and Ad-TIKI2-124, respectively. The injections were repeated every other day for five times, with a total dosage of $1 \times 10^{9} \mathrm{pfu}$ of adenoviruses. 
A

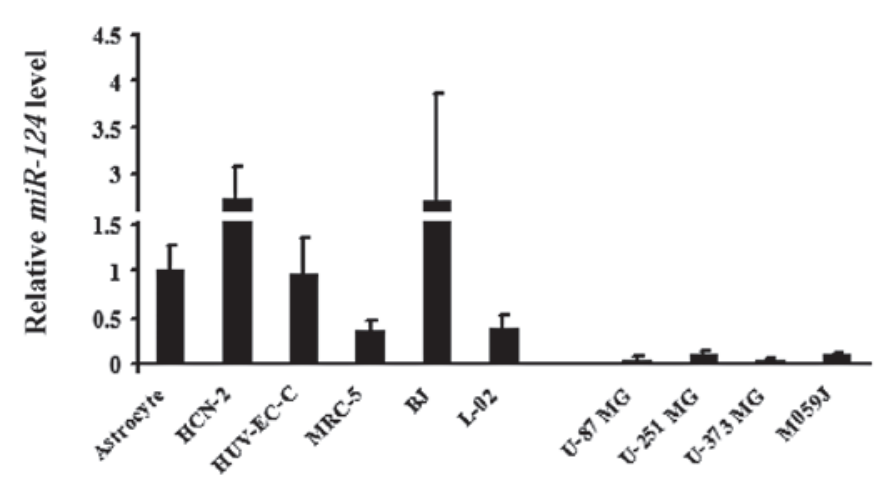

B

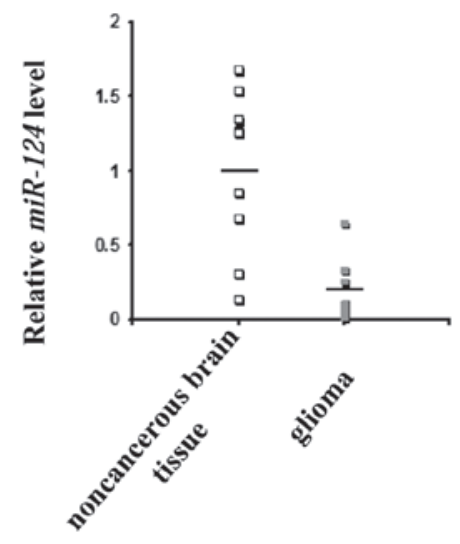

C

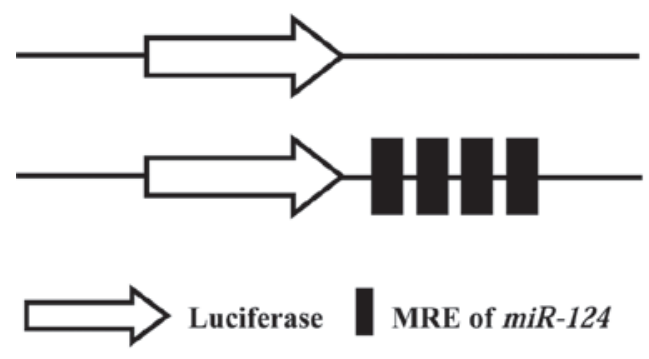

D

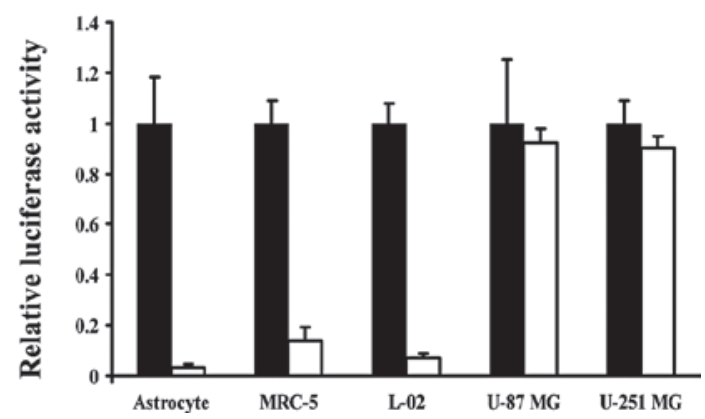

Figure 1. microRNA response element of $m i R-124$ inhibits exogenous gene expression within glioma cells. (A) Expression levels of $m i R-124$ were detected in different cell lines by quantitative polymerase chain reacion. (B) $m i R-124$ abundance was quantified in primary glioma specimens and their corresponding noncancerous tissues $(\mathrm{n}=9)$. (C) Schematic structure of the luciferase vectors. (D) Luciferase activity was detected in glioma and normal cell lines. Data are presented as the mean \pm standard deviation. MRE, microRNA response element; miR-124, microRNA-124. Black bars, psiCheck2; white bars, psiCheck2-124.

The tumor diameter was measured by periodic measurements using calipers and the volume was calculated using the following formula: Tumor volume $\left(\mathrm{mm}^{3}\right)=$ maximal length $(\mathrm{mm}) \mathrm{x}$ [perpendicular width $(\mathrm{mm})]^{2} / 2$. The tumor weights were measured when the mice were sacrificed. Immunohistological staining was used to determine the localization of $\beta$-catenin in the sections of established glioma.

Statistical analysis. Each experiment was performed at least three times. All values are expressed as the mean \pm standard deviation, and compared with the unpaired two-tailed t-test. $\mathrm{P}<0.05$ was considered to indicate a statistically significant difference.

\section{Results}

MREs of miR-124 mediate glioma-specific TIKI2 expression. The first aim of the present study was to construct a glioma-specific expression vector for exogenous genes, including TIKI2. $m i R-124$ has been shown to be underexpressed in glioma cells as compared with noncancerous brain tissue; therefore, the MREs of miR-124 were used to restrict exogenous gene expression within glioma cells for subsequent experiments. The levels of $m i R-124$ were confirmed to be downregulated in glioma cell lines (Fig. 1A) and primary glioma from patients (Fig. 1B) by qPCR assays. Subsequently, a luciferase reporter assay was used to verify the effectiveness of the miR-124 MREs in the regulation of inserted genes (Fig. 1C). The data indicated that the MREs-regulated
psiCheck2 vector expressed luciferase in a glioma-selective fashion (Fig. 1D).

Adenovirus-mediated TIKI2 expression suppresses the activation of the WNT pathway in glioma cells. An adenoviral vector expressing TIKI2 was constructed, which harbored four copies of MREs of miR-124 following the coding region of TIKI2 (Ad-TIKI2-124) (Fig. 2A). The Ad-TIKI2-124, and Ad-TIKI2 and Ad-EGFP control vectors were transduced into U-87 MG and U-251 MG cells. qPCR assays confirmed that TIKI2 was highly expressed in the tested glioma cells infected with both Ad-TIKI2-124 and Ad-TIKI2 (Fig. 2B). Immunoblot analysis of $\beta$-catenin expression revealed that the phosphorylation of $\beta$-catenin was enhanced in Ad-TIKI2-124- and Ad-TIKI2-infected glioma cells, with a reduced stabilization of $\beta$-catenin (Fig. 2C). TOPFlash reporter experiments indicated that the transcriptional activity of transcription factor/lymphoid enhancer-binding factor (TCF/LEF), the major downstream effector in the $\mathrm{WNT} / \beta$-catenin pathway, was potently inhibited in the glioma cells infected with the two distinct TIKI2-expressing adenoviruses (Fig. 2D). The mRNA abundance of the WNT signaling target genes was markedly decreased in U-87 MG and U-251 MG cells upon infection with Ad-TIKI2-124 and Ad-TIKI2 (Fig. 2E).

Ad-TIKI2-124 and Ad-TIKI2 exert high anti-tumor activity on glioma cells. The effects of the TIKI2-expressing adenoviral vector on the biological traits of glioma cells was analyzed. MTT assays indicated that Ad-TIKI2-124 
A

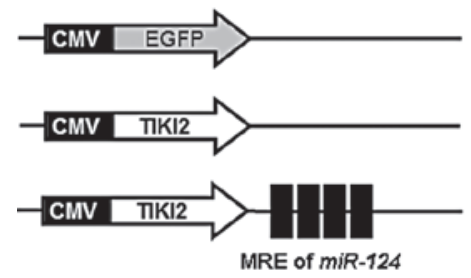

D

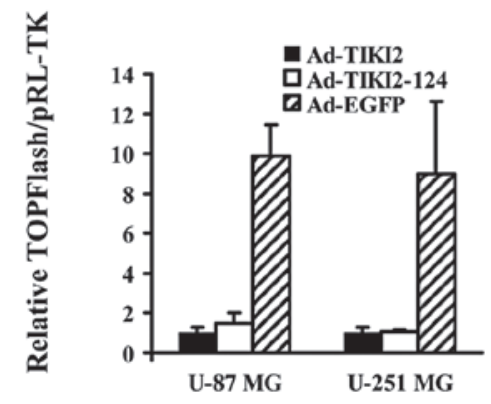

B

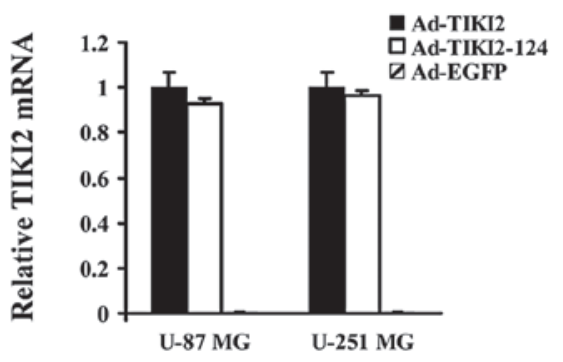

$\mathbf{E}$

Axin2

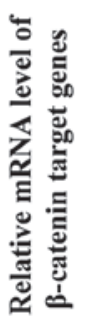

C

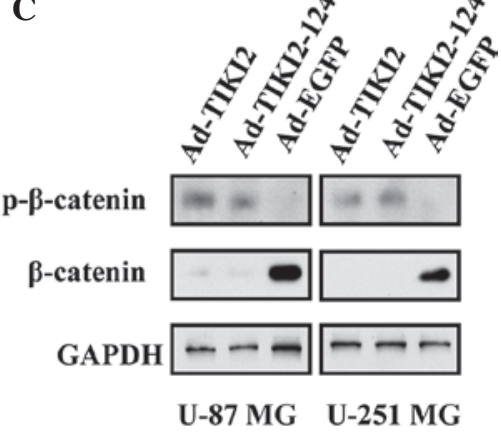

Lgr5

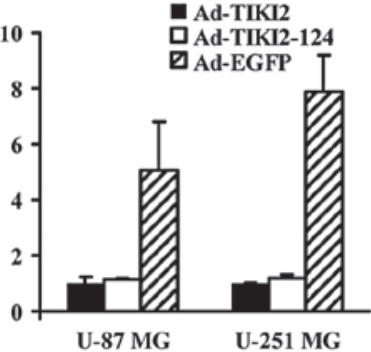

Figure 2. TIKI2 gene expression suppresses the activation of the WNT signaling pathway in glioma cells. (A) Schematic structure of adenoviruses expressing TIKI2. (B) Analysis of TIKI2 mRNA expression in glioma cells infected with different adenoviruses. (C) Immunoblot analysis of the expression levels of phosphorylated and total $\beta$-catenin in glioma cells infected with adenoviruses. (D) Transcription activity of TCF/LEF was determined using TOPFlash reporter assay. (E) mRNA levels of WNT target genes, Axin2 and Lgr5, were assessed in glioma cells following transduction of adenoviral vectors. EGFP, enhanced green fluorescent protein; MRE, microRNA response element; Ad, adenovirus; miR-124, microRNA-124; TCF/LEF, transcription factor/lymphoid enhancer-binding factor.

A

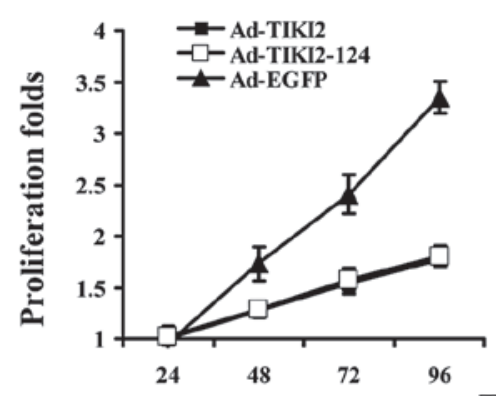

U-251 MG

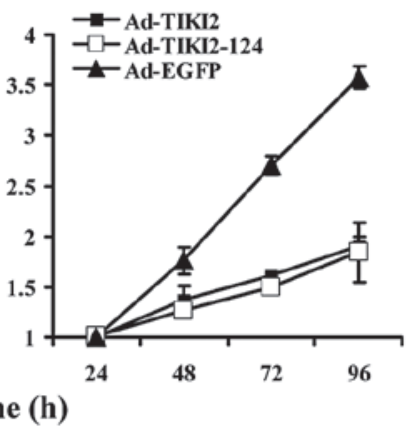

B

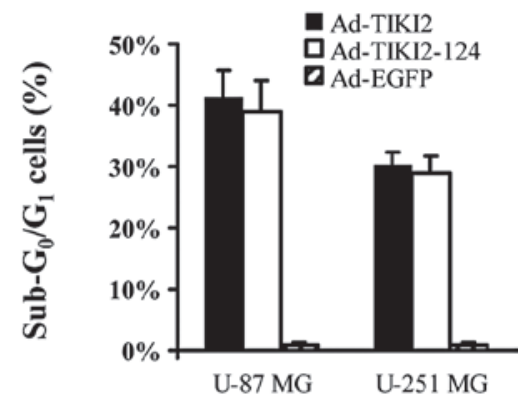

C

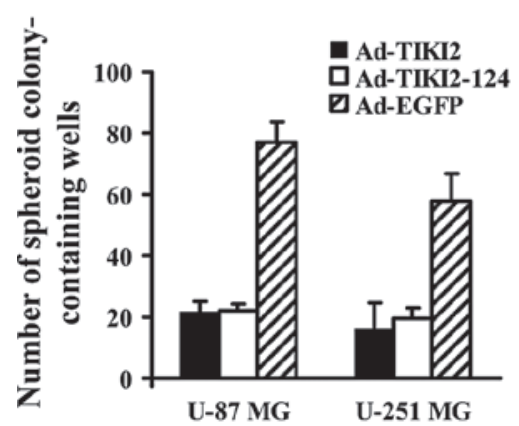

D

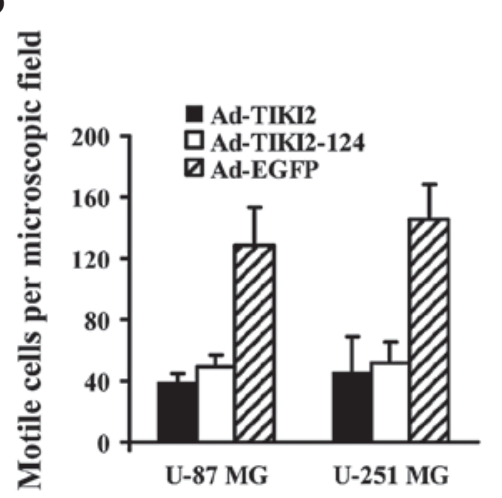

$\mathbf{E}$

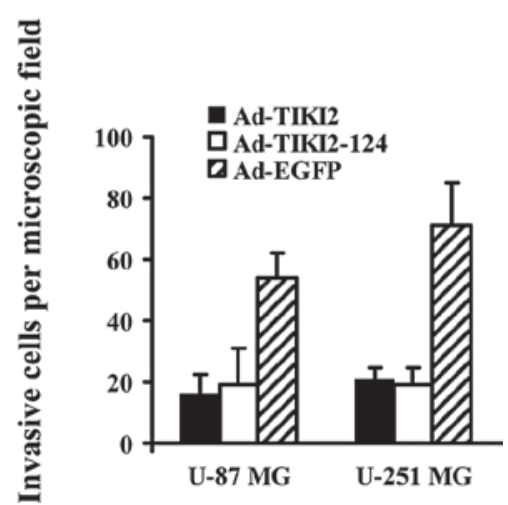

Figure 3. TIKI2 gene expression mediated by adenovirus exerts anti-tumor activity on glioma cells. (A) The proliferation rates of glioma cells infected with adenoviruses were detected by MTT assay. (B) The sub- $\mathrm{G}_{0} / \mathrm{G}_{1}$ population was quantified in Ad-TIKI2- and Ad-TIKI2-124-transduced glioma cells by flow cytometry. (C) The colony numbers of glioma cells were determined following transduction of adenoviral vectors expressing TIKI2 and control vector. (D) The motility and (E) the invasiveness of glioma cells overexpressing TIKI2 were evaluated using a transwell assay. Ad, adenovirus; EGFP, enhanced green fluorescent protein. 

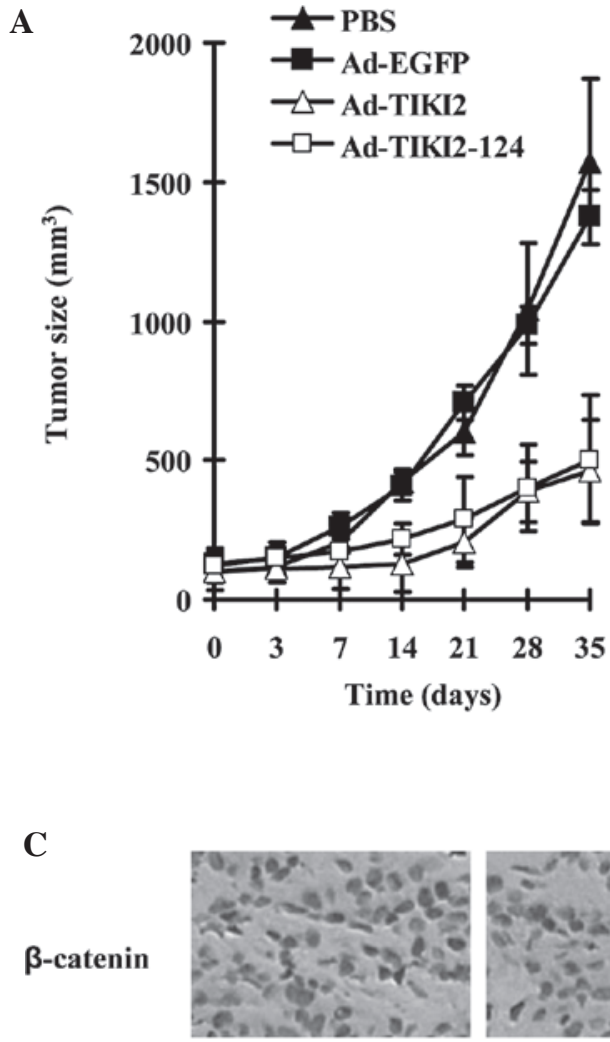

PBS

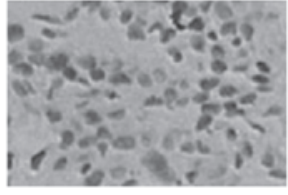

Ad-EGFP

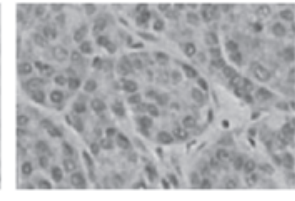

Ad-TIKI2

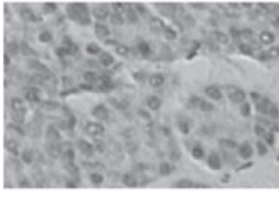

Ad-TIKI2-124

Figure 4. Ad-TIKI2 and Ad-TIKI2-124 reduce the growth of U-87 MG xenografts. (A) Tumor sizes are shown following intratumoral injection of Ad-TIKI2, Ad-TIKI2-124, Ad-EGFP and PBS (n=6). (B) Tumor weight presented graphically as dots. (C) Immunohistological staining was used to localize the expression of $\beta$-catenin in established gliomas in mice. Ad, adenovirus; PBS, phosphate-buffered saline; EGFP, enhanced green fluorescent protein. Magnification, x100.

A

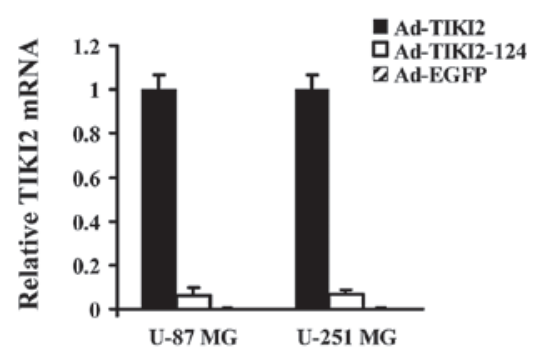

B

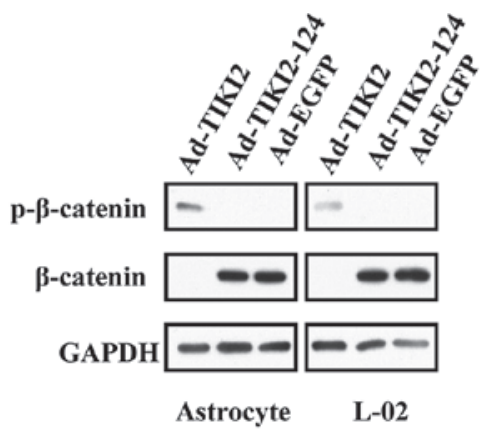

C

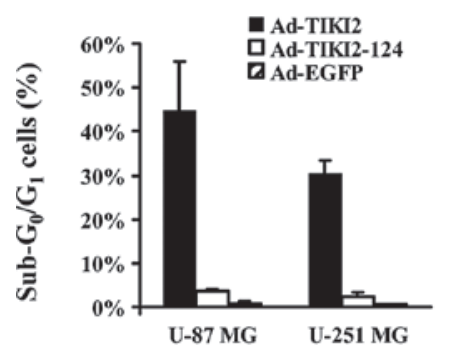

D
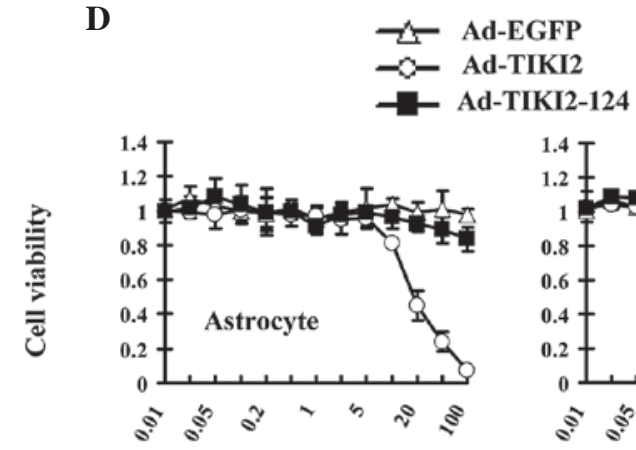

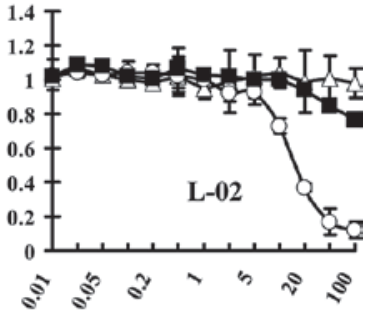

MOIs

Figure 5. TIKI2 does not cause WNT-associated toxicity in normal cells. (A) Expression of TIKI2 mRNA was evaluated in normal cells infected with different adenoviruses. (B) Expression levels of phosphorylated and total $\beta$-catenin were determined by immunoblot assay in normal cells infected with adenoviruses. (C) The sub- $\mathrm{G}_{0} / \mathrm{G}_{1}$ population was quantified in Ad-TIKI2- and Ad-TIKI2-124-transduced normal cells by flow cytometry. (D) The viability of normal cells transduced with adenoviruses was detected by MTT assay. mRNA, microRNA; Ad, adenovirus; EGFP, enahnced green fluorescent protein; p, phosphorylated; MOI, multiplicity of infection. 
and Ad-TIKI2 was able to decelerate the proliferation of glioma cells (Fig. 3A). The determination of the sub- $G_{0} / G_{1}$ subpopulation revealed that TIKI2 expression lead to an increase in the percentage of apoptotic U-87 MG and U-251 MG cells (Fig. 3B). Furthermore, soft agar assays showed that Ad-TIKI2-124 and Ad-TIKI2 infection of glioma cells greatly compromised the colony formation ability, evidenced by a markedly lower number of colonies detected in the group transduced with the two vectors as compared with the group infected with Ad-EGFP (Fig. 3C). Finally, the motility and invasion capacity of glioma cells were found to be highly impaired when TIKI2 expression mediated by the infected adenoviruses occurred (Fig. 3D and E).

Growth of U-87 MG xenograft is suppressed by TIKI2 expression in vivo. The antitumor activity of TIKI2 was further studied in a mouse model. U-87 MG cells were subcutaneously injected into the flanks of mice. When the tumor grew to 8-10 mm, Ad-TIKI2, Ad-TIKI2-124, Ad-EGFP and PBS were intratumorally administrated. The diameter of these tumors was periodically measured using a caliper and their sizes were calculated (Fig. 4A). The weights of U-87 MG cells are presented in Fig. 4B. Immunohistological staining revealed that extranuclear translocation of $\beta$-catenin frequently occurred in the tumors treated with Ad-TIKI2 and Ad-TIKI2-124, while $\beta$-catenin accumulated in the nuclei of Ad-EGFP and PBS-injected tumors (Fig. 4C).

Ad-TIKI2-124 protects normal cells from TIKI2-mediated suppression of WNT pathway. The effect of TIKI2 expression was a major concern in the present study. It was therefore investigated whether TIKI2 is able to affect the activation of WNT signaling in normal cells and induce cytotoxicity. qPCR assays revealed that TIKI2 expression was suppressed in normal cells infected with Ad-TIKI2-124, which may be due to the existence of $m i R-124$ in these cell lines (Fig. 5A). Immunoblot assays indicated that $\beta$-catenin was stabilized in astrocyte and L-02 cells infected with Ad-TIKI2-124 (Fig. 5B), suggesting that WNT signaling was not affected by the treatment of Ad-TIKI2-124. In addition, the percentage of sub- $\mathrm{G}_{0} / \mathrm{G}_{1}$ cells was not altered in Ad-TIKI2-124-transduced normal cells, as compared with Ad-EGFP-infected cells (Fig. 5C). Finally, MTT assays revealed that there was no significant cytotoxicity to normal cells when Ad-TIKI2-124 was added to the cultures (Fig. 5D).

\section{Discussion}

There has been extensive research into glioma gene therapy for many years. A recent phase III clinical trial revealed that adenovirus-mediated herpes simplex virus thymidine kinase (HSV-tk) expression significantly prolonged the survival of glioma patients (13). This encouraging result is expected to attract increasing attention from additional researchers.

The suppressor selected for glioma gene therapy is critical to ensure effective treatment. To date, numerous genes have been trialled for glioma therapy (14). In addition to HSV-tk, key genes in the glioma-specific molecular pathways have additionally been studied for gene therapy. Adenovirus-mediated delivery of calreticulin and melanoma-associated antigen 3 has been shown to inhibit invasion and angiogenesis of U-87 MG glioblastoma cells through suppression of the metastasis-associated pathway (15). TNF-related apoptosis-inducing ligand and arresten expression mediated by adenoviral vectors have also been demonstrated to potently suppress glioma cell growth by targeting its survival pathway (16). Adenovirus-mediated delivery of basic fibroblast growth factor small interfering RNA was found to suppress the activation of signal transducer and activator of transcription 3 signaling, and thus, induce the apoptosis in U-251 MG glioma cells (17).

Aberrant WNT/ $\beta$-catenin signaling has been shown to have a central function in the development of glioblastoma (5). Proliferation, apoptosis and invasion have been identified to be affected by the activation of $\mathrm{WNT} / \beta$-catenin signaling. Various miRNAs and small molecular compounds have been shown to exert anti-glioma activity by suppressing the activation of the WNT pathway (5), suggesting that targeting this pathway may be an effective strategy for glioma gene therapy. The current strategy used to suppress the activation of WNT signaling is by transfection of glioma cells with $\beta$-catenin siRNA (9); however, the therapeutic effect is limited by the low efficiency of transfection.

In the present study, a distinct method to suppress the activation of the WNT pathway was employed. TIKI2, a protease specific for WNT protein inactivation, was inserted into adenoviral vectors for intracellular expression in glioma cells. Subsequent experiments revealed that TIKI2 potently inhibited WNT/ $\beta$-catenin signaling in glioma cell lines.

To improve the biosafety of TIKI2 expression-based gene therapy, MREs of $m i R-124$ were utilized to confer its expression with high selectivity for glioma cells. Previous studies have demonstrated that the application miRNA MREs is able to regulate exogenous gene expression in a glioma-specific fashion (18-21). To further improve the selectivity of gene expression mediated by adenoviral vectors, additional miRNA MREs may be selected for glioma therapy. $m i R-122$, a liver-enriched miRNA, can be used to further reduce the cytotoxicity induced by TIKI2 expression in hepatic cells (21).

In addition to the TIKI gene family, other WNT inhibitors could also be used for glioma gene therapy. Dickkopf WNT signaling pathway inhibitor 1 (DKK1) has been shown to suppress the proliferation of glioma cells, secreted from mesenchymal stem cells of the umbilical cord (22), and a therapeutic effect of DKK1 expression has additionally been verified in gastric cancers (23). Therefore, DKK1 may also be selected as an exogenous gene for MRE-regulated adenoviral vector overexpression in future studies.

Finally, the fiber region of adenoviral vectors can be reconstructed to enhance infectiveness for glioma cells. Currently, a 5/35 chimeric adenovirus that harbors a fused fiber protein from the two different serotypes has been tested for glioma gene therapy (24), and this recombinant adenovirus has been used in gene therapy treatment of a wide range of cancers $(23,12)$. In future experiments, the $5 / 35$ adenoviral vector may be used to express TIKI 2 in order to potently suppress WNT signaling.

In conclusion, a TIKI 2 expressing adenovirus regulated by MREs of miR-124 was investigated for its use in glioma therapy. Experimental data confirmed that this strategy is 
highly effective for glioma treatment and may be promising for clinical application.

\section{References}

1. Reardon DA, Galanis E, DeGroot JF, et al: Clinical trial end points for high-grade glioma: the evolving landscape. Neuro Oncol 13: 353-361, 2011.

2. Recławowicz D, Stempniewicz M, Biernat W and Słoniewski P: Modern approach to WHO grade II glioma classification and treatment - review of the literature. Neurol Neurochir Pol 42: 536-545, 2008

3. Mitlianga PG, Sioka C, Vartholomatos G, et al: p53 enhances the Delta-24 conditionally replicative adenovirus anti-glioma effect. Oncol Rep 15: 149-153, 2006.

4. Wang X, Han L, Zhang A, et al: Adenovirus-mediated shRNAs for co-repression of miR-221 and miR-222 expression and function in glioblastoma cells. Oncol Rep 25: 97-105, 2011.

5. Zhang K, Zhang J, Han L, Pu P and Kang C: Wnt/beta-catenin signaling in glioma. J Neuroimmune Pharmacol 7: 740-749, 2012.

6 . Clevers $\mathrm{H}$ and Nusse R: Wnt/ $\beta$-catenin signaling and disease. Cell 149: 1192-1205, 2012.

7. Kamino M, Kishida M, Kibe T, et al: Wnt-5a signaling is correlated with infiltrative activity in human glioma by inducing cellular migration and MMP-2. Cancer Sci 102: 540-548, 2011.

8. Gotze S, Wolter M, Reifenberger G, Muller O and Sievers S: Frequent promoter hypermethylation of Wnt pathway inhibitor genes in malignant astrocytic gliomas. Int J Cancer 126 2584-2593, 2010.

9. Pu P, Zhang Z, Kang C, et al: Downregulation of Wnt2 and beta-catenin by siRNA suppresses malignant glioma cell growth Cancer Gene Ther 16: 351-361, 2009.

10. Zhang X, Abreu JG, Yokota C, et al: Tiki1 is required for head formation via Wnt cleavage-oxidation and inactivation. Cell 149: $1565-1577,2013$.

11. Xia H, Cheung WK, Ng SS, et al: Loss of brain-enriched miR-124 microRNA enhances stem-like traits and invasiveness of glioma cells. J Biol Chem 287: 9962-9971, 2012.

12. He X, Liu J, Yang C, et al: 5/35 fiber-modified conditionally replicative adenovirus armed with p53 shows increased tumor-suppressing capacity to breast cancer cells. Hum Gene Ther 22: 283-292, 2011.
13. WestphalM,Ylä-HerttualaS,MartinJ,etal: Adenovirus-mediated gene therapy with sitimagene ceradenovec followed by intravenous ganciclovir for patients with operable high-grade glioma (ASPECT): a randomised, open-label, phase 3 trial. Lancet Oncol 14: 823-833, 2013.

14. Tobias A, Ahmed A, Moon KS and Lesniak MS: The art of gene therapy for glioma: a review of the challenging road to the bedside. J Neurol Neurosurg Psychiatry 84: 213-222, 2013.

15. Liu XL, Zhao D, Sun DP, et al: Adenovirus-mediated delivery of CALR and MAGE-A3 inhibits invasion and angiogenesis of glioblastoma cell line U87. J Exp Clin Cancer Res 31: 8, 2012.

16. Li X, Mao Q, Wang D, Zhang W and Xia H: A fiber chimeric CRAd vector Ad5/11-D24 double-armed with TRAIL and arresten for enhanced glioblastoma therapy. Hum Gene Ther 23: 589-596, 2012.

17. Liu J, Xu X, Feng X, Zhang B and Wang J: Adenovirus-mediated delivery of bFGF small interfering RNA reduces STAT3 phosphorylation and induces the depolarization of mitochondria and apoptosis in glioma cells U251. J Exp Clin Cancer Res 30: $80,2011$.

18. Bo Y, Guo G and Yao W: MiRNA-mediated tumor specific delivery of TRAIL reduced glioma growth. J Neurooncol 112: 27-37, 2013.

19. Zhao Y, Li Y, Wang L, et al: microRNA response elements-regulated TRAIL expression shows specific survival-suppressing activity on bladder cancer. J Exp Clin Cancer Res 32: 10, 2013.

20. Liu J, Ma L, Li C, et al: Tumor-targeting TR AIL expression mediated by miRNA response elements suppressed growth of uveal melanoma cells. Mol Oncol 2013.

21. Ma L, Liu J, Shen J, et al: Expression of miR-122 mediated by adenoviral vector induces apoptosis and cell cycle arrest of cancer cells. Cancer Biol Ther 9: 554-561, 2010.

22. Ma S, Liang S, Jiao H, et al: Human umbilical cord mesenchymal stem cells inhibit C6 glioma growth via secretion of dickkopf-1 (DKK1). Mol Cell Biochem 2013.

23. Wang B, Liu J, Ma LN, et al: Chimeric 5/35 adenovirus-mediated Dickkopf-1 overexpression suppressed tumorigenicity of CD $44^{+}$gastric cancer cells via attenuating Wnt signaling. J Gastroenterol 48: 798-808, 2013.

24. Hoffmann D, Meyer B and Wildner O: Improved glioblastoma treatment with Ad5/35 fiber chimeric conditionally replicating adenoviruses. J Gene Med 9: 764-778, 2007. 